\title{
Muerte súbita debida a cardiotoxicidad aguda inducida por antraciclinas
}

\section{Orlando D. Navarro-Ulloa a,b,c,*, Luis A. Barranco-Camargo ${ }^{d}$, Sandra P. Jurado-López ${ }^{d}$, Carmen I. Zabala-Carballo ${ }^{d}$ y Luis E. Giraldo-Peniche ${ }^{e}$}

\author{
a Posgrado de Especialidades Médicas. Cardiología. Universidad del Sinú, Seccional Cartagena, Cartagena, Colombia \\ b Dirección Científica Instituto de Cardiología del Caribe, Cartagena, Colombia \\ c Servicio de Cardiología. Clínica Cartagena del Mar S.A.S., Cartagena, Bolívar, Colombia \\ d Posgrado de Neurología clínica, Universidad del Sinú, seccional Cartagena, Cartagena, Colombia \\ e Posgrado de Medicina interna. Universidad del Sinú, seccional Cartagena, Cartagena, Colombia
}

Recibido el 20 de noviembre de 2016; aceptado el 2 de julio de 2017

Disponible en Internet el 11 de noviembre de 2017

\author{
PALABRAS CLAVE \\ Muerte súbita \\ cardiaca; \\ Fibrilación \\ ventricular; \\ Función ventricular \\ izquierda; \\ Ecocardiografía; \\ Radicales libres
}

\begin{abstract}
Resumen Antraciclinas como la doxorrubicina, así como anticuerpos monoclonales, como el trastuzumab, y agentes alquilantes, como la ciclofosfamida, son compuestos muy útiles como quimioterapia citotóxica al reducir en forma significativa la mortalidad relacionada con el cáncer. Sin embargo, su potencial cardiotoxicidad es un efecto adverso mayor que puede presentarse en cualquier momento de su administración o posterior a la misma, en especial cuando se usan combinados. La toxicidad cardiovascular por doxorrubicina suele ser dependiente de dosis e irreversible, mientras la ocasionada por trastuzumab no lo es. Se han encontrado cambios electrocardiográficos habituales durante la administración de quimioterapia, independiente de la dosis acumulada; a estos cambios agudos se les ha dado poca importancia, aunque pueden suceder hasta en el $40 \%$ de los pacientes. A pesar de la aparición documentada de arritmias tanto en humanos como en modelos animales, la muerte súbita cardiaca durante o inmediatamente después de la infusión de quimioterapia no está bien descrita. Se presenta el caso de un adulto joven sin antecedentes cardiovasculares, con linfoma no-Hodgkin y corazón con imagen ecocardiográfica muy sugestiva de infiltración linfomatosa del ventrículo izquierdo, quien desarrolla alteraciones del ritmo cardiaco que condicionan muerte súbita tras la infusión endovenosa lenta de doxorrubicina y trastuzumab.

(c) 2017 Sociedad Colombiana de Cardiología y Cirugía Cardiovascular. Publicado por Elsevier España, S.L.U. Este es un artículo Open Access bajo la licencia CC BY-NC-ND (http:// creativecommons.org/licenses/by-nc-nd/4.0/).
\end{abstract}

\footnotetext{
* Autor para correspondencia.

Correo electrónico: onu1954@hotmail.com (O.D. Navarro-Ulloa).
} 


\section{KEYWORDS}

Cardiac sudden

death;

Ventricular

fibrillation;

Left ventricular

function;

Echocardiography;

Free radicals

\section{Sudden death due to anthracycline-induced acute cardiotoxicity}

Abstract Anthracyclines, such as doxorubicin, and monoclonal antibodies, such as trastuzumab, are compounds of wide clinical use as cytotoxic chemotherapy as they significantly reduce cancer-related mortality. However, the toxicity is a major adverse effect of these agents, which may occur at any time in their administration or afterwards, especially when used in combination. Cardiovascular toxicity by doxorubicin is dose-dependent and irreversible, while that caused by trastuzumab is not. Common electrocardiographic changes have been found during the administration of chemotherapy, independent of the cumulative dose, but these acute changes have been given little importance, although they may occur up to $40 \%$ of patients. Despite documented evidence of arrhythmias in both human and animal models, sudden cardiac death during or immediately after the infusion of chemotherapy is not well described. This paper describes a young adult with no history of cardiovascular disease, with non-Hodgkinś lymphoma and a heart image very suggestive of left ventricle lymphomatous infiltration. The patient developed heart rhythm disturbances that lead to sudden death after slow intravenous infusion of doxorubicin.

() 2017 Sociedad Colombiana de Cardiología y Cirugía Cardiovascular. Published by Elsevier España, S.L.U. This is an open access article under the CC BY-NC-ND license (http:// creativecommons.org/licenses/by-nc-nd/4.0/).

\section{Introducción}

Las antraciclinas (doxorrubicina, daunorrubicina, epirrubicina e idarrubicina) son las drogas de primera línea para el tratamiento de los pacientes con leucemias agudas, linfomas, sarcomas de tejidos blandos y algunos tumores sólidos como el cáncer de mama. Sin embargo, su efectividad antineoplásica se oscurece por su potencial cardiotoxicidad, al punto, que las enfermedades cardiovasculares derivadas del efecto cardiotóxico de la quimioterapia, se consideran, junto a la aparición de una segunda neoplasia, como las principales causas de muerte en los sobrevivientes del cáncer ${ }^{1}$. Según el Cardiac Review and Evaluation Committee, la cardiotoxicidad se define como cualquier enfermedad cardiovascular derivada del uso de medicamentos onco-hematológicos, que debe sospecharse cuando estos pacientes presentan una o más de las siguientes condiciones:

- Miocardiopatía con disminución de la función sistólica ventricular izquierda en forma global o más severa en el septum.

- Síntomas de falla cardiac.

- Signos asociados a falla cardiaca como S3, taquicardia o ambos.

- Disminución de al menos 5\% de la fracción de eyección ventricular izquierda (FEVI) con valores menores al 55\% o signos y síntomas de falla cardiaca, o disminución de al menos $10 \%$ en la FEVI con valores menores al $55 \%$ sin la presencia de signos o síntomas ${ }^{2}$.

Teniendo en cuenta que una FEVI en el límite normal bajo (50-55\%) incrementa el riesgo de cardiotoxicidad en pacientes tratados con antraciclinas o trastuzumab, la Sociedad Americana de Ecocardiografía y la Sociedad Europea de Imágenes Cardiovasculares acordaron 53\% como el umbral normal bajo de la FEVI ${ }^{1,3}$. El corazón puede afectarse de manera directa por lesión estructural o indirecta por trastornos trombogénicos o hemodinámicos. Las formas de presentación son: aguda o subaguda cuando aparece desde el inicio del tratamiento hasta dos semanas después de terminarlo, y crónica cuando se desarrolla después de un año de la quimioterapia; es crónica temprana si se manifiesta durante el primer año posterior al tratamiento, y crónica tardía cuando aparece años después, que pueden ser hasta $40^{4}$. En la forma aguda se han documentado diversas arritmias como taquicardia sinusal, extrasístoles ventriculares y supraventriculares y fibrilación auricular, además de cambios en el electrocardiograma como prolongación del QTc, disminución transitoria de la FEVI y pericarditis. La forma crónica, es dosis-dependiente y acumulativa, manifestándose como disfunción ventricular, miocardiopatía dilatada y falla cardiaca ${ }^{2,4}$.

Los mecanismos implicados en la cardiotoxicidad por quimioterapia son multifactoriales: generación de radicales libres oxigenados (ROS), daños mitocondriales estructurales y funcionales, alteraciones en la homeostasis del calcio y del hierro, y daños en la expresión genética ${ }^{2,4-8}$.

Se describe el caso de un adulto joven con linfoma no-Hodgkin y corazón con imagen ecocardiográfica de infiltración linfomatosa del ventrículo izquierdo, quien iniciando el esquema de quimioterapia, presentó muerte súbita tras la infusión endovenosa lenta de doxorrubicina.

\section{Caso}

Paciente masculino, de 25 años de edad, de raza negra, estudiante universitario, sin comorbilidades previas, quien consultó por fiebre de predominio vespertino hasta $39^{\circ} \mathrm{C}$, asociada a tos seca ocasional, astenia, adinamia y adenopatías generalizadas de un mes de evolución.

Al examen físico: estado nutricional adecuado; adenomegalias en regiones temporal y parietal izquierdas, cervicales, axilares e inguinales bilaterales, sin otras alteraciones. Con la sospecha de síndrome linfoproliferativo, fue valorado por 
Hematología decidiéndose realizar biopsias de ganglios y medula ósea y ampliar estudios complementarios para definir extensión de la enfermedad.

El hemograma, las funciones renal y hepática, el ionograma, el calcio iónico y sérico, el proteinograma, el ácido úrico y el urocultivo fueron normales; los resultados de la PCR, VSG, VIH, anticuerpos para hepatitis fueron negativos; el VDRL no reactivo. De igual manera, $\beta 2$ microglobulina e IgM e IgG para leptospira y citomegalovirus negativas; en tanto que IgG positiva e IgM negativa para dengue e IgG positiva e IgM negativa para Epstein-Barr. La LDH levemente aumentada, pero 25 días después presentó un aumento progresivo, así como de la enzima alanino aminotransferasa.

La ecografía abdominal mostró hepatomegalia leve, mientras la radiografía de tórax y las tomografías de cráneo simple, tórax y abdomen estuvieron dentro de los parámetros normales.

El ecocardiograma documentó ventrículo izquierdo con engrosamiento transmural heterogéneo de las paredes latero posterior y anterior de $22 \mathrm{~mm}$ en segmentos mediales y $25 \mathrm{~mm}$ en región apical con función diastólica normal, contractilidad normal con función sistólica normal y FEVI de 64\% (Teicholz) y 63\% (Simpson biplano); derrame pericárdico pequeño sin taponamiento (fig. 1A).

Con la biopsia ganglionar, la inmunohistoquímica y el informe ALK (protein kinasa de linfoma de células grandes anaplásicas), Hematología consideró linfoma no Hodgkin de células T anaplásico estadio ıv por múltiple compromiso en piel, índice pronóstico internacional [IPI: 3 (intermedio alto)], índice pronóstico para linfoma de células T [PIT: 1 (grupo 2)]. Se decidió tratamiento con protocolo CHOEP (ciclofosfamida, vincristina, doxorrubicina, etopósido, prednisona), primer ciclo prioritario con superficie corporal (SC) $1,8 \mathrm{~m}^{2}$ : doxorrubicina $81 \mathrm{mg}$, vincristina $2 \mathrm{mg}$ y ciclofosfamida $1120 \mathrm{mg}$ endovenoso el día 1; etopósido $162 \mathrm{mg}$ endovenoso del día 1 al 3; prednisona $50 \mathrm{mg} 2$ veces al día vía oral del día 1 al 5; pegfilgrastim $6 \mathrm{mg}$ subcutáneo el día 4; ondansetron $16 \mathrm{mg}$ endovenoso prequimioterapia y $8 \mathrm{mg}$ vía oral cada 8 horas por 5 días; dexametasona $8 \mathrm{mg}$ endovenoso prequimioterapia y esomeprazol $20 \mathrm{mg}$ vía oral día.

Al tercer día de iniciar la quimioterapia presentó muerte súbita, con ritmo inicial de taquicardia ventricular, sin pulso, con posterior ritmo de fibrilación ventricular durante toda la reanimación cardiopulmonar, con respuesta a los 30 minutos, y requerimiento de desfibrilación en 7 ocasiones. Fue trasladado a la unidad de cuidados intensivos (UCl) donde se consideró cardiotoxicidad aguda por terapia CHOEP, particularmente por antraciclinas (doxorrubicina). A pocas horas del evento se hizo nuevo ecocardiograma con una diferencia de 28 días respecto al anterior; se halló ventrículo izquierdo con adelgazamiento del septum interventricular y grosor normal de las paredes restantes y función diastólica normal; hipoquinesia global con disfunción sistólica moderada, FA 17\% y FEVI 36\% (Teicholz) y 32\% (Simpson biplano), con deterioro significativo con relación a estudio previo; sin datos ecocardiográficos de tromboembolia pulmonar. En los ecocardiogramas posteriores al inicio de la quimioterapia se observó desaparición casi total de la infiltración linfomatosa del ventrículo izquierdo (fig. 1B). El strain $2 \mathrm{D}$ por speckle tracking evidenció compromiso difuso con GLS - 8,6\%, alteración marcada en las curvas de contractilidad segmentaria y mejoría evidente en los exámenes consecutivos de los días $2(-14,1 \%), 4(-16,4 \%), 9(-16,8 \%)$ y $22(-18,7 \%)$ (fig. 2). La evolución de la FEVI por Teicholz y Simpson para los mismos días fue: 2 (62\% - 50\%), 4 (60\% $50 \%), 9(64 \%-65 \%)$ y $22(71 \%-73 \%)$.

Durante su hospitalización en UCl presentó signos de shock cardiogénico, con falla cardiaca aguda, taquicardia sinusal y un episodio de taquicardia ventricular monomorfa con imagen de bloqueo de rama izquierda observada en el monitor cardiaco. Se muestran trazados electrocardiográficos previos y posteriores a la desfibrilación (fig. 3). La troponina fue positiva $1 \mathrm{u} / \mathrm{l}$, la CPK total $2.563 \mathrm{u} / \mathrm{l}$, la CPK MB 56 u/l. Se inició manejo con vasoactivos (noradrenalina), betabloqueantes (metoprolol) y antiarrítmico (amiodarona). Al séptimo día del inicio de la quimioterapia se evidenció pancitopenia. Se retiró sedo-analgesia para extubación, y durante el despertar se hizo evaluación neurológica que fue satisfactoria. Posteriormente, por evolución favorable se retiraron vasoactivos y se trasladó a sala general.

\section{Discusión}

Los factores de riesgo para la cardiotoxicidad inducida por antraciclinas son factores genéticos, dosis acumulativas, sexo femenino y falla renal. Los factores de riesgo compartidos con el trastuzumab son: edad $<15$ y $>65$ años, hipertensión arterial, diabetes, cardiopatía isquémica, FEVI en rango normal bajo (50-55\%) previa al tratamiento, historia de falla cardiaca o disfunción cardiaca relacionada con quimioterapia, combinación de quimioterapia y radioterapia torácica; la obesidad $\left(\mathrm{MVI}>30 \mathrm{~kg} / \mathrm{m}^{2}\right)$ y el estilo de vida sedentario se relacionan más con cardiotoxicidad por trastuzumab ${ }^{1,2,8}$.

Según el grado de riesgo para cardiotoxicidad las drogas antineoplásicas se clasifican así:

- Riesgo alto: antraciclinas, ciclofosfamida y trastuzumab.

- Riesgo moderado: docetaxel, pertuzumab, sunitinib, sorafenib.

- Riesgo bajo: bevacizumab, dasatinib, imatinib, y lapatinib ${ }^{1}$.

Entre las manifestaciones cardiovasculares de origen tóxico están: falla cardiaca con disfunción sistólica ventricular (antraciclinas, trastuzumab, inhibidores de tirosin quinasa, ciclofosfamida, bevacizumab), hipertensión arterial (bevacizumab), las arritmias (antraciclinas y taxanes), la hipotensión arterial (etopóxido, alemtuzumab, cetuzumab, rituximab e interleuquina 2), isquemia miocárdica (5-fluoracilo, capecitabina, taxanes, alcaloides, bevacizumab), fenómenos tromboembólicos y pericarditis ${ }^{2}$.

La cardiotoxicidad puede presentarse en diferentes formas: la tipo 1, mediante el efecto similar a los antraciclínicos o "efecto antraciclina", que puede ser aguda, dosis independiente, transitoria, y por hipersensibilidad tipo 1; 0 la forma crónica, dosis dependiente acumulativa e irreversible. La cardiotoxicidad tipo 2 es similar a la de trastuzumab o "efecto trastuzumab" con lesión reversible por no ocasionar daño ultraestructural en los miocitos.

En la cardiotoxicidad tipo ।, el estrés oxidativo explica el principal mecanismo fisiopatológico y se basa en el daño que ocasionan los agentes radicales libres oxigenados (ROS) 


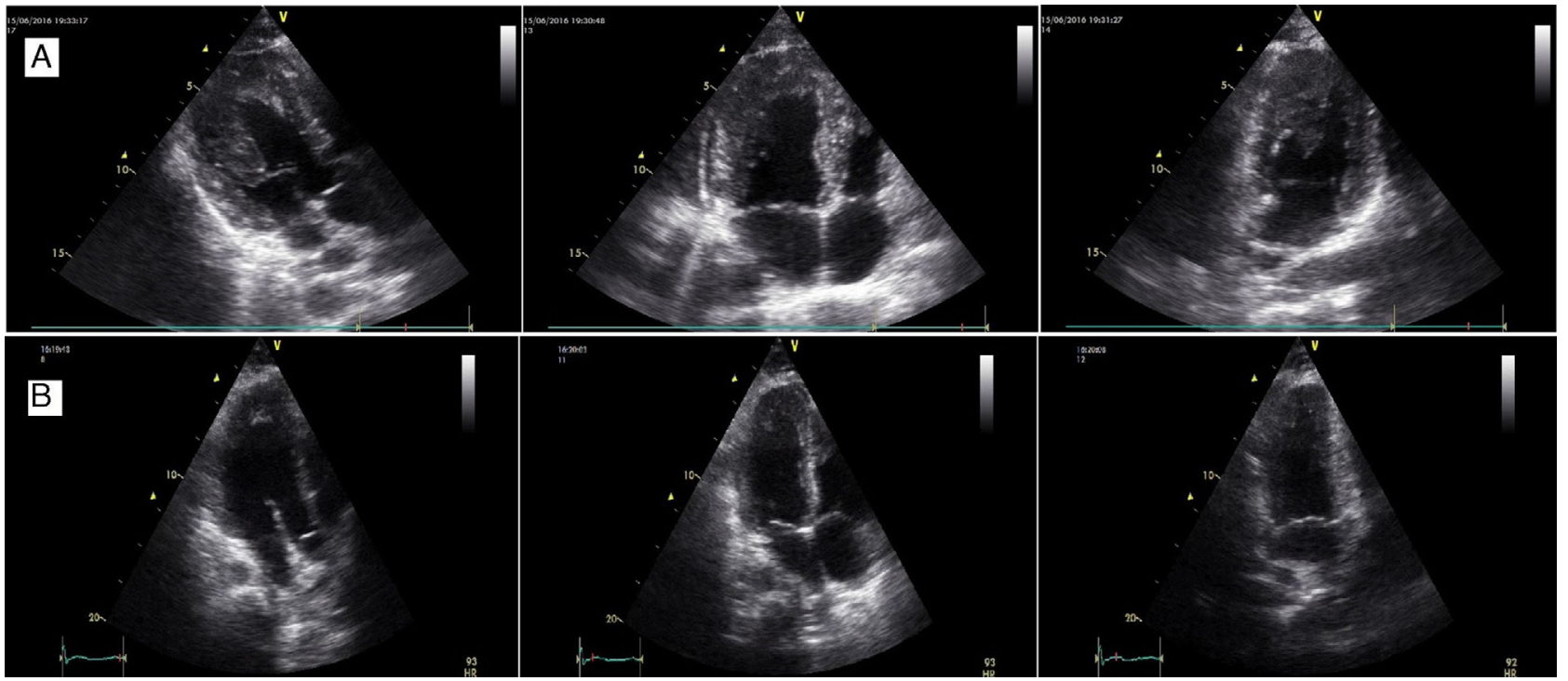

Figura 1 Imágenes obtenidas mediante ecocardiograma transtorácico en proyecciones de 3, 4 y 2 cámaras. A. Arriba: Ecocardiograma previo a la quimioterapia que muestra el engrosamiento del ventrículo izquierdo, en especial de las paredes posterior, lateral y anterior, con densidad ecográfica heterogénea y bordes irregulares, compatible con infiltración linfomatosa en paciente joven con linfoma no-Hodgkin difuso, quien presentó arritmias ventriculares que condujeron a taquicardia ventricular, fibrilación ventricular y muerte súbita durante la infusión intravenosa lenta de doxorrubicina. B. Abajo: Imágenes posteriores al inicio de la quimioterapia que muestran la reducción marcada del tumor. Los linfomas metastásicos al ventrículo izquierdo son muy raros.
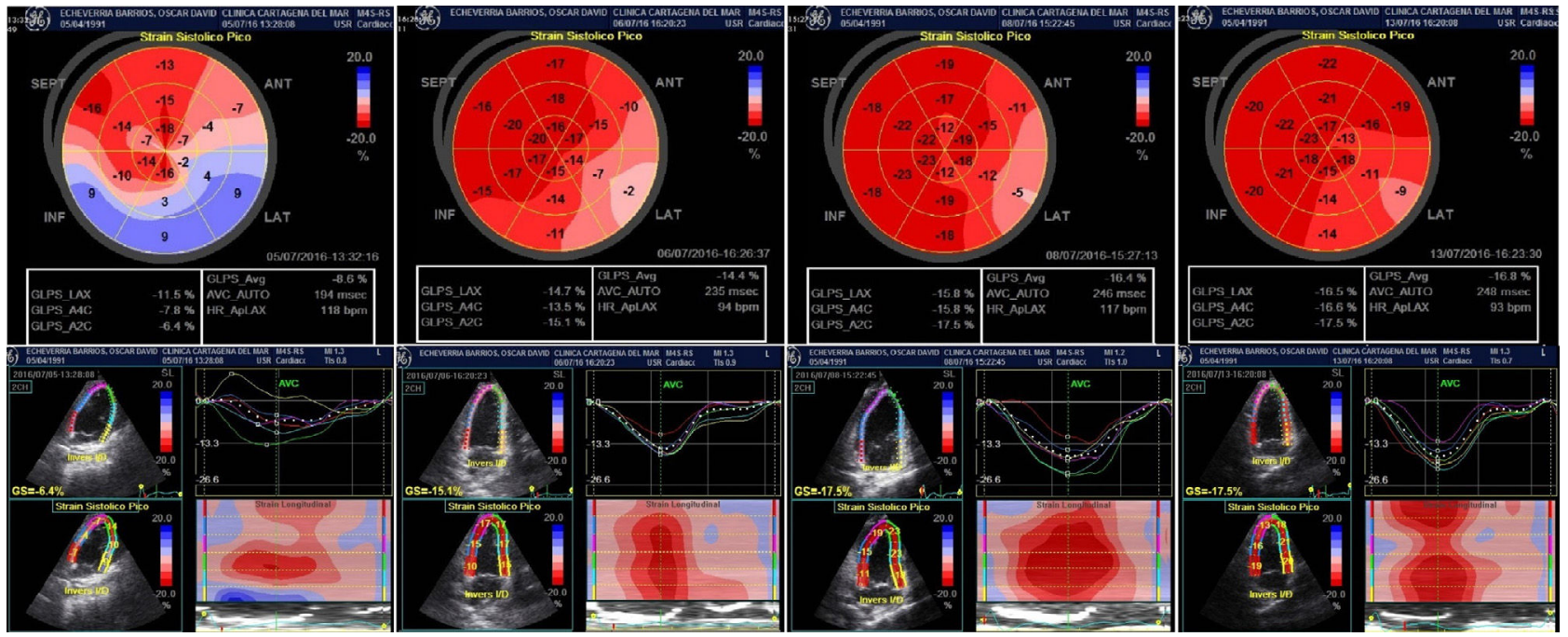

Figura 2 Imágenes de strain 2D por speckle tracking, adquiridas mediante ecocardiogramas transtorácicos en un paciente joven con linfoma no-Hodgkin e infiltración linfomatosa del ventrículo izquierdo, quien presentó cardiotoxicidad aguda inducida por doxorrubicina, manifestada con arritmias ventriculares y muerte súbita. La primera corresponde al día del evento y muestra el compromiso difuso con GLS - 8,6\%, alteración marcada en las curvas de contractilidad segmentaria y mejoría evidente en los exámenes consecutivos de los días $2(-14,1 \%), 4(-16,4 \%), 9(-16,8 \%)$ y $22(-18,7 \%)$.

por reducción del grupo quinona en el anillo $B$ de las antraciclinas, formando un radical semiquinona que al oxidarse dan lugar ROS como el superóxido $\left(\mathrm{O}^{2-}\right)$ con la generación de peróxido de hidrógeno $\left(\mathrm{H}_{2} \mathrm{O}_{2}\right)$ que interactúa con el miocardio y desequilibra el balance entre antioxidantes y sustancias proinflamatorias, que predisponen a lesión celular al reducir la glutatión peroxidasa involucrada en la función antioxidante. La formación de un complejo de hierro férrico con la doxorrubicina, cataliza esta reacción e incrementa los ROS, convierte más hierro ferroso en férrico, lisa las membranas celulares y el retículo endoplasmático mediante un proceso de peroxidación lipídica en cadena de los fosfolípidos de las membranas celulares y subcelulares, reduce la actividad de la $\mathrm{Ca}^{2+}$-ATPasa sarcoplasmática y disminuye el calcio intracelular y, por consiguiente, la contractilidad. Las citoquinas inflamatorias provocan la liberación de histamina, el factor de necrosis tumoral alfa (FNT-alfa) y la interleuquina 2 (IL2), y por ende, el desarrollo 

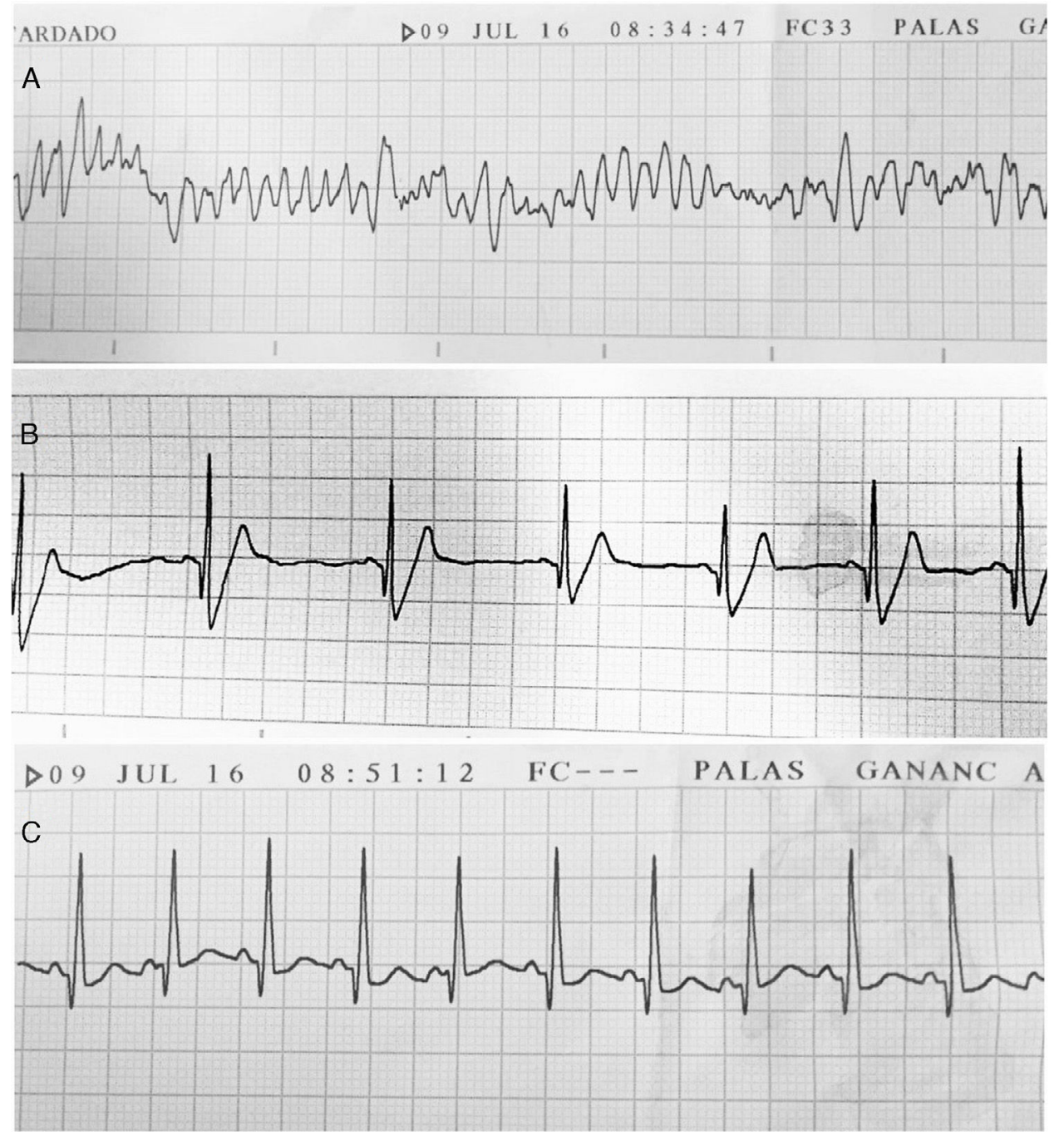

Figura 3 Trazados electrocardiográficos durante el episodio de muerte súbita por cardiotoxicidad aguda por doxorrubicina. A: Fibrilación ventricular. B. Posterior a la cardioversión eléctrica aparece el ritmo sinusal a 75 lpm y QRS ancho. C: Taquicardia sinusal a 140 lpm y QRS estrecho.

de miocardiopatía dilatada y la disfunción beta-adrenérgica. Las topoisomerasas también se han implicado en la toxicidad por antraciclínicos; la doxorrubicina ejerce su papel antineoplásico al formar un complejo ternario con una de las isoenzimas denominada Top2-doxorrubicina-ADN y alterar la expresión genética al inhibir la topoisomerasa 2, esencial en la separación de las hebras durante la replicación del $A D N$. El resultado final es la muerte del miocito, asociada a deprivación del crecimiento y compromiso de la capacidad de reparación debido a la interrupción de la angiogénesis e inducción de la apoptosis, ${ }^{2,4}$.

En la cardiotoxicidad tipo ॥, el agente biológico trastuzumab se une al receptor 2 tirosin quinasa transmembrana del factor de crecimiento epidérmico (HER2) internalizándolo. Este receptor funciona como proto-oncogen regulando el crecimiento celular, sobreexpresándose en el $25 \%$ de los cánceres de mama como marcador de mal pronóstico. Este HER2 en el corazón se une a la neurregulina transformándose en HER2/neu, que previene la apoptosis de los miocitos; el efecto antiproliferativo del trastuzumab se produce en las células que expresen este receptor, que, al ser inhibido, aceleran la apoptosis y disminuyen la recuperación funcional que conduce a la disfunción ventricular y a la falla cardiaca ${ }^{2}$.

Mientras la incidencia de toxicidad cardiovascular de cada uno de estos dos grupos de medicamentos por separado representa el 3 al 7\%, la combinación la eleva al $27 \%$ en especial en pacientes mayores de 80 años, hipertensos o con cardiopatía isquémica ${ }^{2,4}$.

La cardiotoxicidad por inhibidores de tirosin quinasa como imatinib, desatinib y nilotinib se basa en la disfunción mitocondrial por generación de proteínas no plegadas que estimulan las vías de respuesta al estrés del retículo endoplasmático, activando las vías PERK e IRE 1 (protein kinase $\mathrm{R}$-like endoplasmic reticulum kinase, inositol-requiring kinase 1), los cuales son receptores protein quinasa transmembrana residentes en el retículo endoplasmático que detectan señales agresoras provenientes de las proteínas no plegadas, y ocasionan autofosforilación y apoptosis por la activación de caspasas y proteínas homólogas C/BEP $(\mathrm{CHOP})^{2}$. 
Otro medicamento importante es la ciclofosfamida, un agente alquilante cuyo mecanismo de acción se basa en provocar daño al ADN y suele utilizarse para el manejo de algunas neoplasias hematológicas y en los trasplantes; es capaz de inducir cardiotoxicidad aguda que puede manifestarse desde alteraciones electrocardiográficas sutiles hasta miocardiopatía incluso fatal. Su efecto es dosis -dependiente y es más frecuente a dosis superiores a $200 \mathrm{mg} / \mathrm{kg}$. Sus efectos fatales se han reportado hasta en $11 \%$ de los casos. Las manifestaciones pueden aparecer dentro de las primeras semanas del tratamiento y podría considerarse un factor que predispone al empeoramiento de la cardiotoxicidad de las antraciclinas ${ }^{2}$.

En la actualidad no se cuenta con escalas de riesgo predictivas de cardiotoxicidad que hayan sido validadas y a pesar de compartir muchos factores de riesgo con las enfermedades cardiovasculares, las escalas tradicionales subestiman el riesgo para toxicidad cardiovascular. Sin embargo, el consenso de expertos en Cardio-OncoHematología, recomienda usar el SCORE, antes de iniciar la quimioterapia, como una herramienta que ayudaría a identificar a los pacientes en riesgo de desarrollar cardiotoxicidad bajo ( $<1 \%)$, moderado $(\geq 1 \mathrm{y}<5 \%)$, alto $(\geq 5 \mathrm{y}<10 \%)$ y muy alto $(\geq 10 \%)^{1}$. Los pacientes con enfermedades cardiovasculares, diabetes más factores de riesgo cardiovascular y/o microalbuminuria o falla renal con filtración glomerular $<30 \mathrm{ml} / \mathrm{min}$, tendrán el riesgo más alto, independiente del puntaje alcanzado, hecho que obligaría a extremar las medidas de protección.

Existen múltiples métodos para detectar la cardiotoxicidad inducida por quimioterapia. La FEVI es un parámetro predictivo en la evaluación inicial de la función sistólica y durante la quimioterapia, pero tiene la limitante que el deterioro de la FEVI es una manifestación tardía del daño miocárdico irreversible. La evaluación ecocardiográfica de la función diastólica puede detectar alteraciones de la función ventricular previas a la disminución de la FEVI, el estudio de la función ventricular derecha mediante el desplazamiento sistólico del anillo de la tricúspide (TAPSE $\geq 1,6 \mathrm{~cm}$ ), la velocidad sistólica del anillo (onda $S^{\prime} \geq 10 \mathrm{~cm} / \mathrm{s}$ ) y el cambio fraccional del área por $2 \mathrm{D}$ (FAC $\geq 35 \%)$. El eco $2 \mathrm{D}$ strain por speckle tracking mide la deformación miocárdica sistólica cuando los miocitos se acortan en las dimensiones longitudinal y circunferencial (strain negativo) y se engruesan y alargan en dirección radial (strain positivo). El strain longitudinal global (GLS), el strain radial y el strain rate poseen evidencia de superioridad respecto a la FEVI en la evaluación del paciente con quimioterapia, con base en los siguientes aspectos: estudia mejor la mecánica ventricular y posee mayor capacidad de predicción de la falla cardiaca y mortalidad; es útil en la insuficiencia cardiaca aguda; es fiable debido a la poca variabilidad entre observadores; tiene capacidad de detección precoz subclínica de la cardiotoxicidad y permite detectar alteraciones sutiles de la función del ventrículo izquierdo ${ }^{7,8}$. Los valores normales mínimos para el GLS son: $-16,7 \%$ para hombres y $-18,5 \%$ para mujeres. Una caída del $15 \%$ en el GLS con relación al basal indicaría disfunción ventricular izquierda subclínica, mientras que la ausencia de alteración o reducciones $<8 \%$ la descartarían ${ }^{8}$.

Existen múltiples estudios que comprueban que el strain es un potente predictor de los eventos cardiacos y parece ser mejor que la FEVI como parámetro para evaluar a los pacien- tes con insuficiencia cardiaca aguda ${ }^{9-12}$. Se han incluido otros parámetros como el strain radial miocárdico sistólico máximo, marcadores ecocardiográficos de la función diastólica, la fracción aminoterminal del propéptido natriurético cerebral (NT-proBNP) y la troponina I cardiaca (cTnl) y se ha concluido que la reducción del GLS entre la situación basal y la evaluación realizada a los tres meses y la troponina detectable a los tres meses, fueron factores predictivos de carácter independiente respecto a la aparición de cardiotoxicidad a los seis meses ${ }^{12}$. Así, pese a que el strain parece ser más sensible para detectar cardiotoxicidad temprana, a la fecha no existe una guía para iniciar cardioprotección específica con los antioxidantes eficaces para preservar la función cardiaca ${ }^{13}$, o para cambiar la antraciclina convencional por otra menos tóxica de tipo liposomal, o suspender o disminuir la dosis de quimioterapia, cuando se detectan los signos subclínicos de disfunción miocárdica ${ }^{14,15}$. Esperar el desarrollo de cardiotoxicidad manifiesta, no constituye una buena estrategia, si se tiene en cuenta que la falla cardiaca secundaria a miocardiopatía inducida por antraciclinas tiene una mortalidad a los dos años tan alta como del $60 \%{ }^{16}$.

Este caso resulta interesante toda vez que muestra cómo el strain complementa al examen bidimensional, e incrementa su valor pronóstico, diagnóstico y seguimiento en los pacientes que reciben quimioterapia. Es necesario enterar a los médicos intensivistas, hematólogos y oncólogos que, ante la sospecha de cardiotoxicidad aguda, el ecocardiograma strain con speckle tracking puede evaluar en forma más precisa la mecánica ventricular y es más sensible y específico para detectar de manera oportuna la lesión miocárdica, su extensión y gravedad y monitorizar su evolución mediante el valor del GLS, las curvas de contractilidad segmentaria y el mapa polar, tal como lo muestran las imágenes del caso expuesto, adicional a que lo hace en tiempo real, en la cabecera del paciente, a bajo costo y no es invasivo.

Sobresale en el caso presentado, el esquema de tratamiento con la combinación de doxorrubicina, trastuzumab y ciclofosfamida y el hallazgo poco frecuente de la infiltración linfomatosa del ventrículo izquierdo, evidenciado en el ecocardiograma prequimioterapia, la cual desapareció tras el inicio de la misma y que por generación masiva de ROS, explicaría, en gran medida, la severidad de la cardiotoxicidad aguda con muerte súbita, en un paciente joven sin factores de riesgo cardiovascular.

\section{Conclusión}

La falla cardiaca es la complicación crónica más frecuente de la quimioterapia, pero aún falta mucha investigación y conocimientos para mitigar este efecto adverso mayor. En cuanto a la cardiotoxicidad aguda, el desconocimiento es superior.

Las enseñanzas de este caso sugieren que los pacientes con infiltración linfomatosa cardiaca deberían ser clasificados en alto riesgo para cardiotoxicidad aguda inducida por antraciclinas, sin otras consideraciones. Ya que el corazón es el órgano más vulnerable a toxicidad, en presencia de infiltración linfomatosa cardiaca, estos pacientes deberían recibir cardioprotección especial y monitorización permanente al inicio del primer ciclo de quimioterapia. Así mismo, el eco strain podría constituir una técnica de alto valor 
clínico y debería incluirse en los protocolos de atención de los pacientes oncológicos. Finalmente, es necesario fomentar los grupos de asistencia e investigación en Cardio-Onco-Hematología, con el fin de profundizar en la fisiopatología de la cardiotoxicidad, validar los marcadores predictivos y las técnicas de detección oportuna, y diseñar estrategias de prevención, cardioprotección, manejo y seguimiento, que hagan más seguros los tratamiento antitumorales y mejoren el pronóstico de los pacientes con cáncer.

\section{Responsabilidades éticas}

Protección de personas y animales. Los autores declaran que para esta investigación no se han realizado experimentos en seres humanos ni en animales.

Confidencialidad de los datos. Los autores declaran que en este artículo no aparecen datos de pacientes.

Derecho a la privacidad y consentimiento informado. Los autores declaran que en este artículo no aparecen datos de pacientes.

\section{Financiación}

Recursos propios.

\section{Conflicto de intereses}

Ninguno.

\section{Bibliografía}

1. López-Fernández T, Martín A, Santaballa A, Montero A, García R, Mazón P, et al. Cardio-Onco-Hematology in Clinical Practice, Position Paper and Recommendations. Rev Esp Cardiol. 2017;70:474-86.

2. Velásquez CA, González M, Berrouet MC, Jaramillo N. Cardiotoxicidad inducida por la quimioterapia desde las bases moleculares hasta la perspectiva clínica. Rev Colomb Cardiol. 2016;23:104-11.

3. Plana JC, Galderisi M, Barac A, Ewer MS, Ky B, Scherrer-Crosbie $M$, et al. Expert Consensus for multimodality imaging evaluation of adult patients during and after cancer therapy: a report from the ASE and the EACVI. J Am Soc Echocardiogr. 2014;27:911-39.

4. Slamon DJ, Leyland-Jones B, Shak S, Fuchs H, Paton V, Bajamonde $\mathrm{A}$, et al. Use of chemotherapy plus a monoclonal antibody against her2 for metastatic breast cancer that overexpresses her2. N Engl J Med. 2001;344:783-92.

5. Klaassen CD, Casarett LJ, Doull J. Casarett \& Doull's toxicology: the basic science of poisons. $8^{\text {th }}$. ed New York: McGraw-Hill Education; 2013. p. 1454, editors.

6. Espinosa E, Zamora P, Feliu J, Santiago A. Cardiac dysfunction secondary to anthracyclines. En: Plana JC, López T, Gómez JJ, García MA, editores. Cardio-Oncology. Madrid: Sociedad Española de Cardiología y CTO Editorial, SL; 2015.

7. Khakoo AY, Liu PP, Force T, Lopez-Berestein G, Jones LW, Schneider J, et al. Cardiotoxicity due to cancer therapy. Tex Heart Inst J Tex Heart Inst St Lukes Episcop Hosp Tex Child Hosp. 2011;38:253-6.

8. Lotrionte M, Biondi-Zoccai G, Abbate A, Lanzetta G, D’Ascenzo F, Malavasi V, et al. Review and meta-analysis of incidence and clinical predictors of anthracycline cardiotoxicity. Am J Cardiol. 2013;112:1980-4

9. Plana JC. La quimioterapia y el corazón. Rev Esp Cardiol. 2011;64:409-15.

10. Plana JC. Imaging techniques in the detection of sub-clinical left ventricle dysfunction. En: Plana JC, López T, Gómez JJ, García MA, editores. Cardio-Oncology. Madrid: Sociedad Española de Cardiología y CTO Editorial, SL; 2015.

11. Cho GY, Marwick TH, Kim HS. Global 2-dimensional strain as a new prognosticator in patients with heart failure. J Am Coll Cardiol. 2009;54:618-24.

12. Sawaya H, Sebag IA, Plana JC, Januzzi JL, Ky B, Cohen V, et al. Early detection and prediction of cardiotoxicity in chemotherapy treated patients. Am J Cardiol. 2011;107:1375-80.

13. Kalam K, Marwick TH. Role of cardioprotective therapy for prevention of cardiotoxicity with chemotherapy: A systematic review and meta-analysis. Eur J Cancer. 2013;49:2900-9.

14. Negishi K, Negishi T, Haluska BA, Hare JL, Plana JC, Marwick $\mathrm{TH}$. Use of speckle strain to assess left ventricular responses to cardiotoxic chemotherapy and cardioprotection. Eur Heart J Cardiovasc Imaging. 2014;15:324-31.

15. Zamorano JL, Lancellotti P, Rodriguez D, Aboyans V, Asteggiano R, Galderisi M, et al. 2016 ESC Position Paper on cancer treatments and cardiovascular toxicity developed under the auspices of the ESC Committee for Practice Guidelines. Eur Heart J. 2016;37:2768-801.

16. Swain SM, Whaley FS, Ewer MS. Congestive heart failure in patients treated with doxorubicin: A retrospective analysis of three trials. Cancer. 2003;97:2869-79. 